\title{
Spectrum Occupancy and Interference Model based on Network Experimentation in Hospital
}

\author{
Lorenzo Mucchi, Senior Member IEEE, Risto Vuohtoniemi, Member IEEE, Hasnain Virk, \\ Andrea Conti, Senior Member IEEE, Matti Hämäläinen, Senior Member IEEE, Jari Iinatti, Senior Member IEEE, \\ Moe Z. Win, Fellow IEEE
}

\begin{abstract}
Emerging healthcare radio technologies are designed to operate in the $2.4 \mathrm{GHz}$ industrial, scientific and medical (ISM) band. Since both standardized (Bluetooth and Wi-Fi) and non-standardized (proprietary) devices use the same frequency band, the aggregate interference may significantly affect the performance of medical wireless systems. This paper characterizes the spatiotemporal spectrum occupancy and proposes models for the aggregate interference in hospital environments. In particular, time-frequency and cluster-based statistical models for the aggregate interference are developed based on network experimentation. The proposed models enable the design of wireless networks for e-health applications and medical services.
\end{abstract}

Index Terms-Wireless networks, medical ICT, e-health, interference, spectrum occupancy, network experimentation.

\section{INTRODUCTION}

Radio frequency spectrum is a finite resource coordinated by regulatory bodies around the globe. In particular, regulatory bodies in different countries allocate frequency bands for healthcare [1]. This includes the sub-GHz ISM band, the $2.4 \mathrm{GHz}$ ISM band, and the $3-10 \mathrm{GHz}$ band [1]. In such bands, there are multiple sources of interference such as that generated by Wi-Fi, Bluetooth, ZigBee, and cordless phones all operating in the $2.4 \mathrm{GHz}$ band.

The increasing deployment of wireless technologies in hospitals will significantly contribute to the electromagnetic pollution, and, consequently, the interference will be a limiting factor of their succesful operation. Spectral occupancy (SO),

Manuscript submitted March 11, 2019; revised September 29, 2019 and February 24, 2020; accepted April 13, 2020. Date of publication Month Day, 2020; date of current version Month Day, 2020. This research was supported, in part, by the European Union's Horizon 2020 research and innovation programme under Grants 871249 and 872752, KAUST Sensor Research Initiative Award OSR-2015-SENSORS-2700, Academy of Finland's 6Genesis Flagship Grant 318927 and ETSI SmartBAN. The material in this paper was presented, in part, at the 2014 Bodynets, London, UK The associate editor coordinating the review of this article and approving it for publication was S. Pollin. (Corresponding author: Lorenzo Mucchi).

Lorenzo Mucchi is with the Department. of Information Engineering and CNIT, University of Florence, 50139 Florence, Italy (e-mail lorenzo.mucchi@unifi.it).

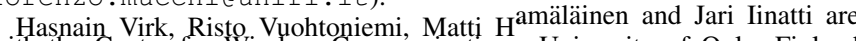
with the Centre for Wireless Communications, University of Oulu, Finland (email: name.surnamedoulu.fi)

Andrea Conti is with the Department of Engineering and CNIT, University of Ferrara, 44122 Ferrara, Italy (e-mail: a.conti@ieee.org).

Moe Z. Win is with the Laboratory for Information \& Decision Systems (LIDS), Massachusetts Institute of Technology, Cambridge, MA 02139, USA (e-mail: moewin@mit.edu).

Color versions of one or more of the figures in this article are available online at http://ieeexplore.ieee.org.

Digital Object Identifier 10.1109/TWC.2020.XXXXXXX which affects the level of interference, provides insights into the coexistence of heterogeneous wireless networks [2]-[10]. Therefore, it is important to determine the spectral occupancy (SO) in the $2.35-2.50 \mathrm{GHz}$ band to characterize the aggregate interference. Dynamic spectrum access relies on accurate evaluation of the SO. Different SO metrics, namely channel occupancy $(\mathrm{CO})$, frequency band occupancy (FBO), and spectrum resource occupancy (SRO), have been considered respectively to represent the utilization of a channel, frequency-band or spectrum resource over a period of time.

In [11], a measurement campaign in the $2.4 \mathrm{GHz}$ ISM band was conducted to determine the SO in an university environment. In [12], experimental results on the coexistence between Bluetooth and Wi-Fi in the ISM band are reported. In [13], a measurement campaign is carried out to determine the type of interference harming Wi-Fi devices. In [14], experimental results on interference between $\mathrm{Wi}$ Fi and other radio devices (such as microwave ovens) operated on a $2.4 \mathrm{GHz}$ ISM band are described. Measurement campaigns for SO characterization in hospital environments have considered mainly the $2.4 \mathrm{GHz}$ ISM band [15] and were performed over a single day continuously [16] or over multiple days in specific hours [17]. Since the number of active devices can vary significantly from one day to another, especially in a hospital environment, continuous measurements over multiple days are of great importance. In addition to the ISM band, other bands have been proposed for medical information and communication technologies (ICT). In particular, in 2012, the Federal Communications Commission (FCC) set aside $40 \mathrm{MHz}$ of spectrum in the $2.36-2.40 \mathrm{GHz}$ band dedicated specifically for wireless medical devices. Similarly, the European Telecommunications Standard Institute (ETSI) is considering setting aside $16.5 \mathrm{MHz}$ in the $2.4835-2.50 \mathrm{GHz}$ band. A comprehensive study of SO in ISM band and nonISM bands for e-health applications and medical services is still missing in the literature.

This paper characterizes the spatiotemporal SO in the 2.35$2.50 \mathrm{GHz}$ band. In addition to the ISM band, we carried out measurements in the lower $2.35-2.40 \mathrm{GHz}$ band and in the higher $2.4835-2.50 \mathrm{GHz}$ band. ${ }^{1}$ Based on three measurement

\footnotetext{
${ }^{1}$ One of the measurement campaigns was carried out by the ETSI Smart Body Area Networks (SmartBANs) Group [18] and has been reported in [19][22]. While in [23] some preliminary results are reported on the spectrum occupancy, based on a limited measurement campaign, this paper largely extend the measurements campaigns and propose two statistical models of the aggregate interference.
} 
campaigns at Oulu University Hospital, various spatiotemporal SO metrics have been evaluated and two statistical models of the aggregate interference have been proposed. The first model, referred to as the time-frequency (TF) model, describes the aggregate interference plus noise over the entire frequency band and over the whole week. The second model, referred to as cluster-based (CB) model, describes the behaviour of aggregate interference clusters over the whole week at representative frequencies having low, moderate, and high interference. While the TF model is useful for simulating the aggregate interference plus noise, the CB model is useful for generating an aggregate interference.

The contributions of the paper can be summarized as follows:

- three one-week long network experimentation campaigns in a hospital to measure the level of interference activity in the ISM band;

- a statistical analysis of the occupancy of the frequency channels in the ISM band; and

- two statistical models of the aggregate interference in the ISM band.

The proposed models serve as basis for simulating network behavior and designing medical devices (e.g., electrocardiogram sensors) in wireless hospital environments. The findings in the paper suggest how to make effective use of the ISM band for mission-critical medical services.

The reminder of this paper is organized as follows. In Sec. II the evaluation of the SO is discussed, while in Sec. III the measurement campaigns and the equipment are reported. Sec. IV defines the channel occupancy, the frequency band occupancy and the spectrum resource occupancy metrics, while Sec. V describes the experimentation in hospital environments. Sec. VI discusses the statistical models of the aggregate interference, and Sec. VII concludes the paper.

Notation: random variables (RVs) are displayed in sans serif, upright fonts; their realizations in serif, italic fonts. Vectors and matrices are denoted by bold lowercase and uppercase letters, respectively. For example, a RV and its realization are denoted by $\mathrm{x}$ and $x$; a random vector and its realization are denoted by $\mathbf{x}$ and $\boldsymbol{x}$; a random matrix and its realization are denoted by $\mathbf{X}$ and $\boldsymbol{X}$, respectively. Sets and random sets are denoted by upright sans serif and calligraphic font, respectively. For example, a random set and its realization are denoted by $\mathrm{X}$ and $\mathcal{X}$, respectively.

\section{SPECTRUM OCCUPANCY CHARACTERIZATION}

The characterization of SO involves collection and processing of measurement data obtained in different settings. The International Telecommunication Union-Radio Communication Sector (ITU-R) guidelines for SO measurements are reported in [24]-[26]. Following the ITU-R guidelines, the samples collected during each frequency sweep of the entire bandwidth are grouped into regulated channels utilized by wireless systems (e.g., Wi-Fi, Bluetooth, and Zigbee).

The sweeping rate of frequency band affects the characterization of different temporal behaviors of the interference. For example, bursty interference could not be captured by infrequent sweeping of the band as in [27]. It is also important to characterize the behavior of the interference over the entire frequency band without restricting to channels utilized by specific wireless systems. Furthermore, it is crucial to design threshold for determining the SO metrics according to a false-alarm vs. missed-detection tradeoff. In this paper, an iterative algorithm known as median forward consecutive mean excision (FCME) [28], [29] is applied over each sweep to dynamically determine the threshold $\eta_{\text {th }}$ for guaranteeing a desired false-alarm probability $p_{\text {FA }}^{\star}$.

The samples recorded by the spectrum analyzer (SA) in the $2.35-2.50 \mathrm{GHz}$ band are organized into a time-frequency sample matrix

$$
\boldsymbol{S}=\left[\boldsymbol{s}_{1}, \boldsymbol{s}_{2}, \ldots, \boldsymbol{s}_{n}, \ldots, \boldsymbol{s}_{N}\right]
$$

of size $L \times N$, where $L$ and $N$ represent the total number of sweeps and frequency samples in the band, respectively. The $n$th column of $\boldsymbol{S}$ is given by

$$
\boldsymbol{s}_{n}=\left[s_{1, n}, s_{2, n}, \ldots, s_{\ell, n}, \ldots, s_{L, n}\right]^{T}
$$

where the element $s_{\ell, n}$ represents the sample of the received power corresponding to the $\ell$ th sweep at the $n$th frequency. For example, in the second measurement campaign (accident and emergency ward) the SA acquires $K=1200$ frequency samples over the $2.35-2.50 \mathrm{GHz}$ band (i.e., separation between two consecutive frequency samples was $125 \mathrm{kHz}$ ) in a $3 \mathrm{~ms}$ long sweep. The processing time between two consecutive sweeps was $45 \mathrm{~ms}$. The number of sweeps is $L=1250$, which means that an acquisition time of $1 \mathrm{~min}$ is needed to capture a single time-frequency matrix $\boldsymbol{S}$. The total bandwidth under investigation has been divided into 150 channels, $1 \mathrm{MHz}$ wide each. Every channel is thus composed of eight (1200/150) frequency samples.

\section{MEASUREMENT SETTINGS}

Three one-week-long network experimentation campaigns have been undertaken in Oulu University Hospital (OUH) to characterize the SO in the $2.35-2.50 \mathrm{GHz}$ band. ${ }^{2}$ The hospital has more than 1000 beds and is equipped with state-of-the-art medical equipment, several ambulatory bays, and a helipad. ${ }^{3}$ To capture the spatial behavior of interference activity, three different locations were considered:

1) daily surgery ward;

2) accident and emergency ward; and

3) X-ray and radiology ward.

The experimental setup is depicted in Fig. 1. Measurements were carried out using Agilent E4446A SA connected to a computer using MATLAB Instrument Control Toolbox. To capture the temporal behavior of the interference, the SA collected data continuously for the whole week as the number of active devices and their position vary from day to day during

\footnotetext{
${ }^{2}$ The radiation limitations are regulated in terms of specific absorption ratio (SAR) by national agencies, including Federal Communications Commission, Food and Drug Administration, and International Commission on NonIonizing Radiation Protection. We characterized the interference generated by the hospital's wireless systems without adding intentional radiations.

${ }^{3} \mathrm{~A}$ detailed description of the hospital can be found in [30].
} 


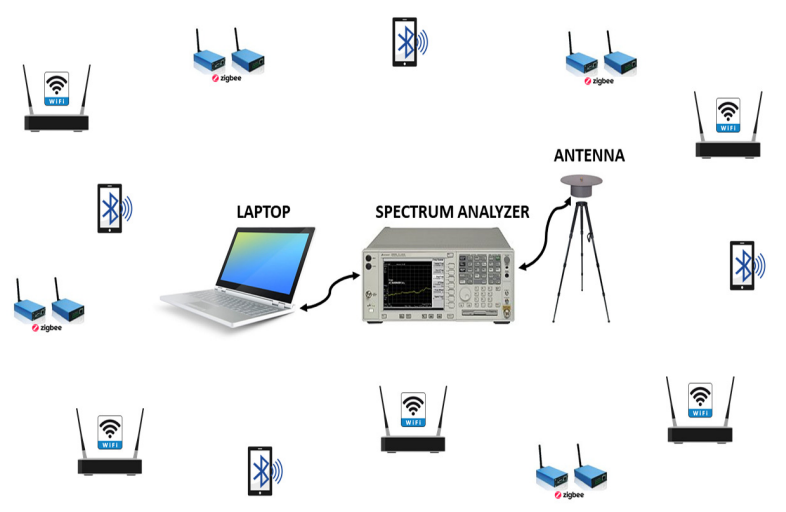

Fig. 1. Equipment and experimental measurement scenario.

the week in a typical hospital. The SA was connected with a $1 \mathrm{~m}$ cable to an omnidirectional, wideband antenna ARA CMA-118/A [31].

The main parameter settings used in the network experimentation are listed in Table I. As proposed by ITU-R [24], the resolution bandwidth was set to $1 / 4(250 \mathrm{kHz})$ of the minimum channel bandwidth $(1 \mathrm{MHz})$ in the second and third measurement campaigns, whereas it was set to $300 \mathrm{kHz}$ in the first measurement campaign due to limitation on data matrix size for MATLAB processing.

\section{SPECTRUM OCCUPANCY METRICS}

The metrics recommended by ITU-R for characterizing the $\mathrm{SO}$ are $\mathrm{CO}, \mathrm{FBO}$, and SRO. Specifically, the CO provides information on the utilization of each channel in the frequency band. The FBO provides information about the utilization of the entire frequency band. The SRO provides information on the utilization of the resources available to a specific wireless system. ITU-R recommends that a channel is marked as occupied if at least $50 \%$ of its frequency samples is above a threshold, which may result in underestimation of true $\mathrm{CO}$. To overcome this issue, we consider the $M \times N$ binary occupancy matrix $\boldsymbol{B}$ with elements

$$
B_{m, n}= \begin{cases}1 & s_{m, n} \geqslant \eta_{\mathrm{th}} \\ 0 & \text { otherwise }\end{cases}
$$

where $s_{m, n}$ is the element of the $M \times N$ matrix obtained by concatenating time-frequency sample matrices $S$ in time. The parameter $M$ represents the number of sweeps per integration time; specifically, $M=4 L$ for the first measurement campaign and $M=L$ for the second and third measurement campaigns. The dynamic threshold $\eta_{\text {th }}$ is determined according to FCME algorithm with $p_{\mathrm{FA}}^{\star}=0.05$.

Recall that the whole $2.35-2.50 \mathrm{GHz}$ band is divided into 150 channels with index set $\mathcal{C}$, each one composed by $N /|\mathcal{C}|$ frequency samples. Let us define the $\mathrm{CO}$ indicator as

$$
B_{m}^{(\mathrm{c})}= \begin{cases}1 & \exists n \in \mathcal{C}^{(c)}: B_{m, n}=1 \\ 0 & \text { otherwise }\end{cases}
$$

TABLE I

PARAMETER SETTINGS FOR THE THREE MEASUREMENTS CAMPAIGNS.

\begin{tabular}{|l|l|l|}
\hline Parameter & Daily Surgery & Emergency/Radiology \\
\hline Frequency band & $2.35-2.50 \mathrm{GHz}$ & $2.35-2.50 \mathrm{GHz}$ \\
Bandwidth & $150 \mathrm{MHz}$ & $150 \mathrm{MHz}$ \\
No. of freq. samples & 1601 & 1200 \\
Resolution bandwidth & $300 \mathrm{kHz}$ & $250 \mathrm{kHz}$ \\
Bin-width & $93.7 \mathrm{kHz}$ & $125 \mathrm{kHz}$ \\
No. of sweeps & 10000 & 1250 per min \\
Sweep time & $2 \mathrm{~ms}$ & $3 \mathrm{~ms}$ \\
Processing time & $22 \mathrm{~ms}$ & $45 \mathrm{~ms}$ \\
Integration time & $4 \mathrm{~min}$ & $1 \mathrm{~min}$ \\
Measurement duration & 1 week & 1 week \\
\hline
\end{tabular}

where $\mathcal{C}^{(c)}$ is the sample index set of the $c$ th channel. The $c$ th $\mathrm{CO}$ probability over the entire measurement campaign can be approximated by averaging $B_{m}^{(\mathrm{c})}$ over all the sweeps as [32]

$$
p_{\mathrm{CO}}^{(c)} \simeq \frac{1}{M} \sum_{m=1}^{M} B_{m}^{(c)} .
$$

The $m$ th sweep FBO probability can be approximated by

$$
p_{\mathrm{FBO}, m} \simeq \frac{1}{N} \sum_{n=1}^{N} B_{m, n} .
$$

The FBO probability over the entire measurement campaign can be approximated by averaging $p_{\mathrm{FBO}, m}$ over all the sweeps as

$$
p_{\mathrm{FBO}} \simeq \frac{1}{M} \sum_{m=1}^{M} p_{\mathrm{FBO}, m} .
$$

The SRO considers the set of channels $\Omega^{(w)}$ used by a specific wireless system $w$. The $m$ th sweep SRO probability for a system $w$ can be approximated by

$$
p_{\mathrm{SRO}, m}^{(w)} \simeq \frac{1}{\left|\Omega^{(w)}\right|} \sum_{c \in \Omega^{(w)}} B_{m}^{(\mathrm{c})} .
$$

The SRO probability for a system $w$ over the entire measurement campaign can be approximated by averaging $p_{\mathrm{SRO}, m}^{(w)}$ over all the sweeps as

$$
p_{\mathrm{SRO}}^{(w)} \simeq \frac{1}{M} \sum_{m=1}^{M} p_{\mathrm{SRO}, m}^{(w)} .
$$

\section{NETWORK EXPERIMENTATION}

We now present the experimentation campaigns in three different wards. The interference measurements are based on the level of energy detected, which depends on the position and type of wireless systems emitting the interfering signals. 


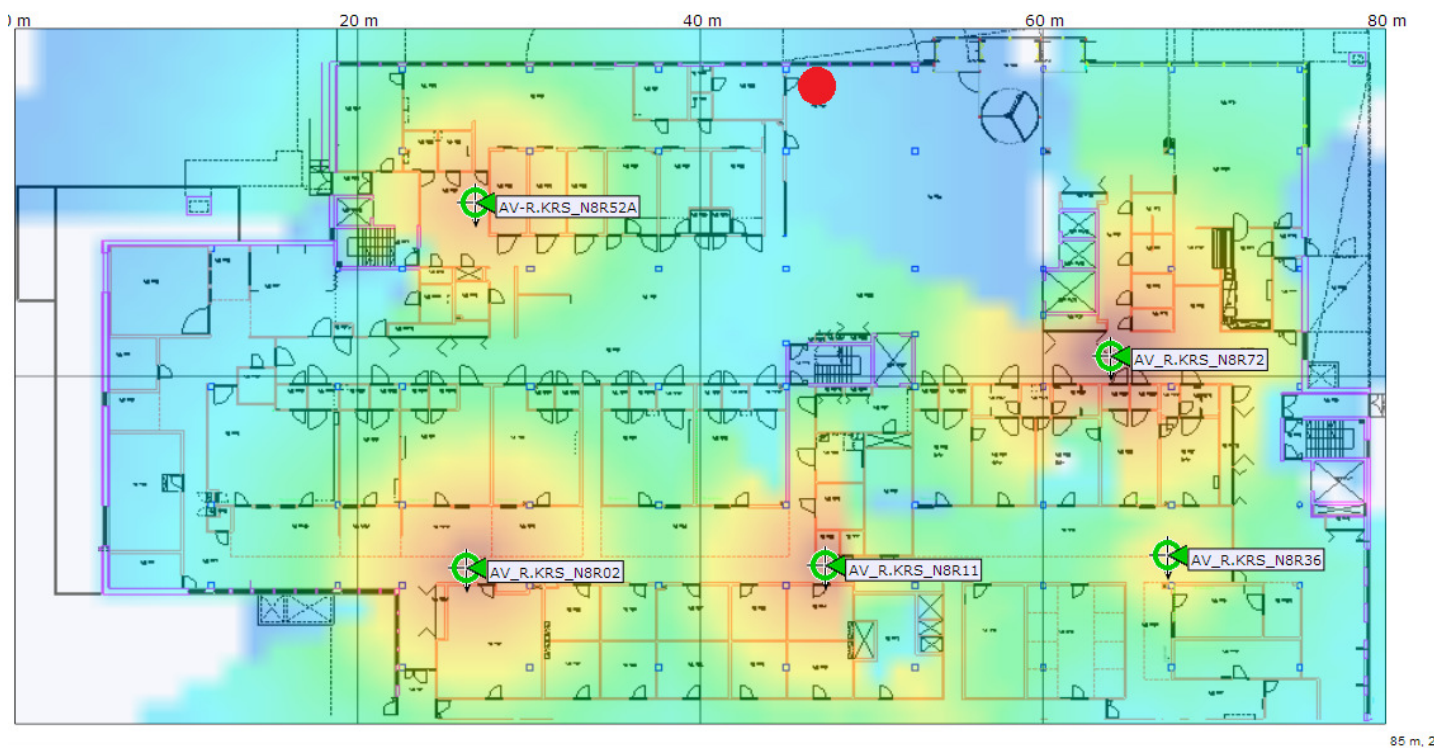

Fig. 2. The floor plan of the daily surgery ward on the ground floor of the hospital. The red point (top center) depicts the location of the spectrum analyzer, while the five green circles depict the fixed Wi-Fi access points. The heatmap of the received power from each access point is also illustrated.

\section{A. Daily surgery ward}

The first measurement campaign was carried out in the daily surgery ward. The floor plan of the daily surgery ward is shown in Fig. 2, where the red dot represents the location of the measurement equipment. The measurement parameters used in this campaign are listed in second column of Table I.

Figs. 3(a) and 3(b) show the CO calculated from measurements on Saturday Dec. 13th and Sunday Dec. 14th, respectively, for the IEEE $802.11 \mathrm{~b} / \mathrm{g}$ channels. Comparing Figs. 3(a) and 3(b) revealed that a high variation of $\mathrm{CO}$ can be seen from one day to another. Although a low interference was observed for channel 11 in most of Sunday Dec. 14th, Fig. 3(b) shows that its $\mathrm{CO}$ was over $50 \%$ for about one hour.

During the measurement campaign it was also observed that the $2.35-2.40 \mathrm{GHz}$ band contain low interference, suggesting that the $2.36-2.40 \mathrm{GHz}$ band allocated by FCC for medical purposes is open for possible exploitation. In Europe, this particular band is already allocated for long term evolution (LTE), which could cause interference to the medical devices. ETSI is currently evaluating the option to reserve the 2.438$2.50 \mathrm{GHz}$ band for medical services or to require medical devices with interference detection and mitigation capabilities.

Fig. 4 shows the CO calculated from measurements on Saturday Dec. 13th for the IEEE 802.11n channels. When compared to Fig. 3(a), it can be observed that interference in IEEE $802.11 \mathrm{n}$ channels is more bursty. This can be attributed to the fact that currently IEEE $802.11 \mathrm{n}$ is less used than IEEE $802.11 \mathrm{~b} / \mathrm{g}$. The small sweep time in our network experimentation, enabled to capture the bursty temporal behavior of the interference in these channels.

Fig. 5 shows the SRO for IEEE $802.11 \mathrm{~b} / \mathrm{g}$ and IEEE $802.11 \mathrm{n}$ channels and FBO calculated from measurements on Sunday Dec. 14th. While the SRO of IEEE $802.11 \mathrm{~b} / \mathrm{g}$ reached $21 \%$, the FBO was $4 \%$. This means that although specific channels can be considered occupied $21 \%$ of the time, $96 \%$ of the entire frequency band is not affected by interference, suggesting that a large portion of the band can be exploited for medical services.

\section{B. Accident and emergency ward}

The second measurement campaign was carried out in the accident and emergency ward. The measurement parameters used in this campaign are listed in third column of Table I.

Fig. 6(a) shows the $\mathrm{CO}$ calculated from measurements over the whole week for the entire $2.35-2.50 \mathrm{GHz}$ band. In addition to IEEE $802.11 \mathrm{~b} / \mathrm{g} / \mathrm{n}$ users in the $2.40-2.47 \mathrm{GHz}$ band, narrowband signals belonging to audio/video systems in bands centered at $2.406 \mathrm{GHz}, 2.427 \mathrm{GHz}$ and $2.490 \mathrm{GHz}$ were observed. It can also be observed that the $2.35-2.40 \mathrm{GHz}$ band contains low interference.

Fig. 6(b) shows the maximum and the mean $\mathrm{CO}$ calculated from measurements over the whole week for the entire 2.35$2.50 \mathrm{GHz}$ band. Although large values of the $\mathrm{CO}$ were observed, most of the channels are scarcely occupied over the entire week, suggesting that a large portion of the band can be exploited for medical services.

Fig. 6(c) shows the SRO in the $2.35-2.50 \mathrm{GHz}$ band $(150$ channels) calculated from measurements over the whole week. A SRO over $15 \%$ was observed on the 4 th, 6 th and 7 th day of the week, while the mean SRO over the whole week was $4.5 \%$. Therefore, $95.5 \%$ of the spectrum resources were unoccupied, on average. Restricting the SRO to ISM band (80 channels) would increase the maximum SRO to $26.38 \%$ and the mean SRO to $8.01 \%$. On the other hand, focusing the $2.36-2.40 \mathrm{GHz}$ band (40 channels) would reduce the maximum SRO to $6.49 \%$ and the mean SRO to $0.1 \%$.

\section{X-ray and radiology ward}

The third measurement campaign was carried out in the $\mathrm{X}$-ray and radiology ward. The X-ray and radiology ward 


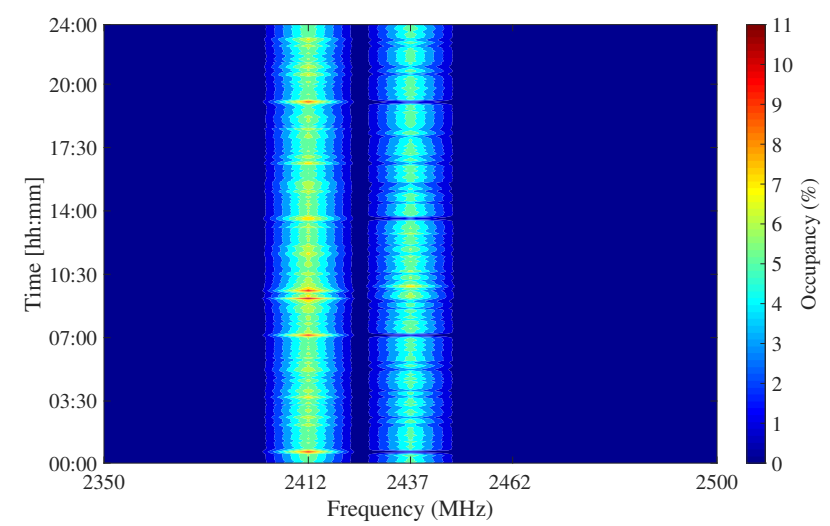

(a) Saturday Dec. 13th.

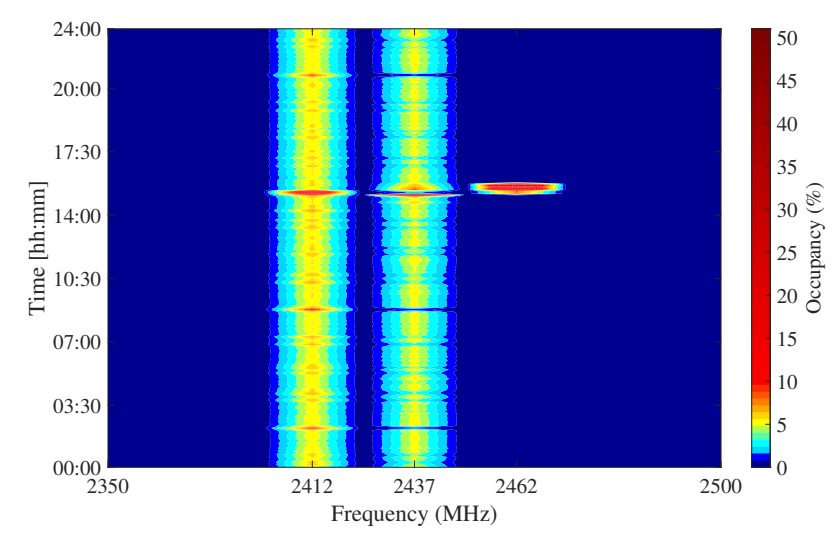

(b) Sunday Dec. 14th.

Fig. 3. CO for channels $1(2.412 \mathrm{GHz}), 6(2.437 \mathrm{GHz})$ and $11(2.462 \mathrm{GHz})$ of the IEEE $802.11 \mathrm{~b} / \mathrm{g}$ (Saturday Dec. 13th and Sunday Dec. 14th). First measurement campaign in daily surgery ward.

contains computer-aided tomography, X-ray, microwave and radio ablation, and diathermy machines. The measurement parameters used in this campaign are listed in third column of Table I.

Fig. 7 shows the $\mathrm{CO}$ calculated from measurements over the whole week for the entire $2.35-2.50 \mathrm{GHz}$ band. Comparing this ward to others revealed that here the $\mathrm{CO}$ was lower and only sporadic interference was detected. This is due to the fact that typical walls in X-ray and radiology ward are shielded, and that this ward is less crowded compared to the others.

\section{STATISTICAL MODELS OF THE INTERFERENCE}

To characterize the aggregate interference in a hospital environment, two statistical models in the $2.35-2.50 \mathrm{GHz}$ band are proposed. The first model describes the aggregate interference (plus noise) over the entire time-frequency intervals; while the second model describes the aggregate interference (without noise) by grouping the interference samples in clusters. Each proposed model is selected among the candidate distributions, as the one that best fits measurement data according to the Bayesian information criterion (BIC) [33] described in the following.

Let $\breve{f}_{\mathrm{x}}(\cdot \mid \boldsymbol{\theta})$ denote a candidate distribution for each element of the random sample with corresponding joint distribution

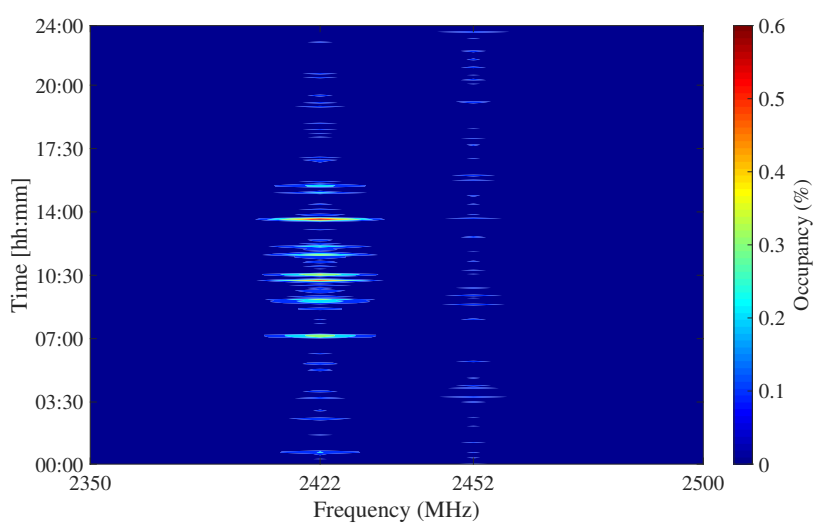

Fig. 4. CO for channels $3(2.422 \mathrm{GHz})$ and $9(2.452 \mathrm{GHz})$ of the IEEE 802.11 n (Saturday Dec. 13th). First measurement campaign in daily surgery ward.

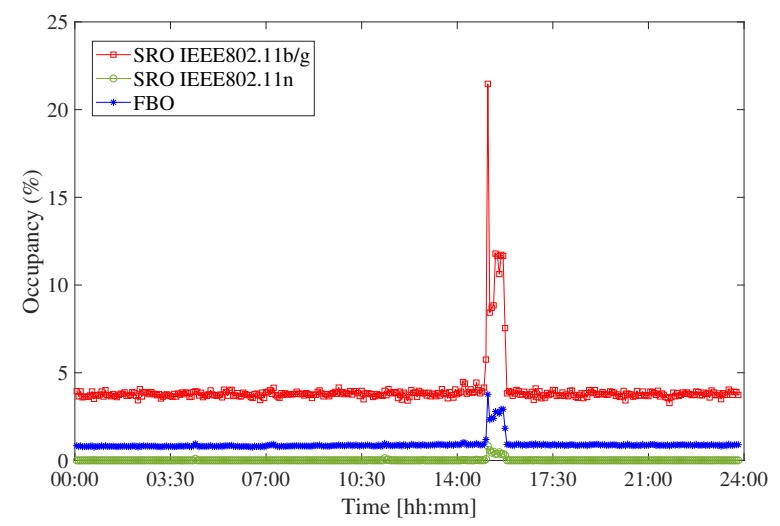

Fig. 5. SRO for IEEE802.11 systems and FBO (Sunday Dec. 14th). First measurement campaign in daily surgery ward.

$\breve{f}_{\mathbf{x}}(\cdot \mid \boldsymbol{\theta})$ and vector parameter $\boldsymbol{\theta}$ of dimension $d$. For a given observed vector $\boldsymbol{x}$ of lenght $n$, the cost associated with $\breve{f}_{\mathbf{x}}(\cdot \mid \boldsymbol{\theta})$ is given by

$$
\zeta_{\mathrm{BIC}}(\boldsymbol{x})=-2 \ln \left(\breve{f}_{\mathbf{x}}(\boldsymbol{x} \mid \hat{\boldsymbol{\theta}})\right)+d \ln (n)
$$

where $\hat{\boldsymbol{\theta}}$ is a maximum likelihood (ML) estimate of $\boldsymbol{\theta}$, i.e.

$$
\hat{\boldsymbol{\theta}}=\arg \max _{\tilde{\boldsymbol{\theta}}} \breve{f}_{\mathbf{x}}(\boldsymbol{x} \mid \tilde{\boldsymbol{\theta}}) .
$$

Among all the candidate distributions, the best fit distribution $f_{\mathrm{x}}(\cdot \mid \boldsymbol{\theta})$ with corresponding joint distribution $f_{\mathrm{x}}(\cdot \mid \boldsymbol{\theta})$ that minimizes the BIC in (10) is selected.

The similarity of the selected distribution with the empirical distribution based on measurement data can be quantified using the Jensen-Shannon divergence (JSD) [34]. Let $p_{1}$ and $p_{2}$ be two probability density function (PDF) representing a $\mathrm{RV} \times$ taking values on a set $\mathcal{X}$, then the JSD is defined as

$$
\mathbb{J}\left\{p_{1} \| p_{2}\right\}=\frac{1}{2} \mathbb{D}\left\{p_{1} \| q\right\}+\frac{1}{2} \mathbb{D}\left\{p_{2} \| q\right\}
$$

where $q=\frac{1}{2}\left(p_{1}+p_{2}\right)$ and $\mathbb{D}\left\{p_{1} \| p_{2}\right\}$ is the Kullback-Leibler divergence, defined as

$$
\mathbb{D}\left\{p_{1} \| p_{2}\right\}=\int_{-\infty}^{\infty} p_{1}(x) \log \left(\frac{p_{1}(x)}{p_{2}(x)}\right) d x .
$$




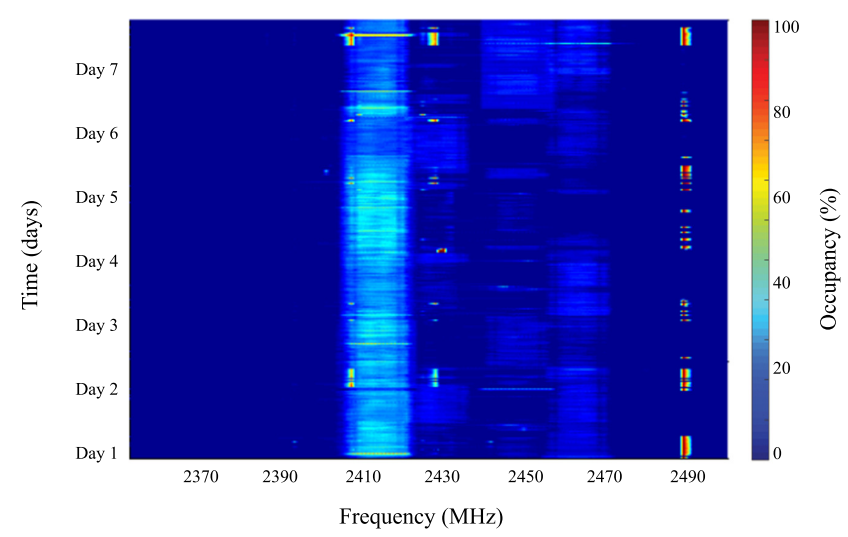

(a) $\mathrm{CO}$ for the entire $2.35-2.50 \mathrm{GHz}$ band.

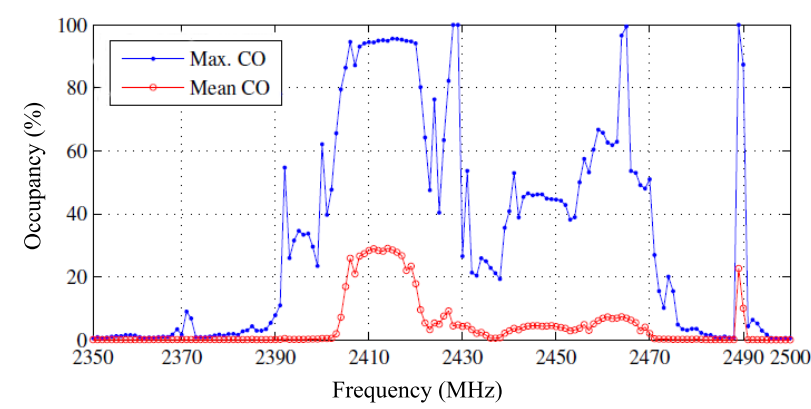

(b) Maximum and mean of the $\mathrm{CO}$ for the entire $2.35-2.50 \mathrm{GHz}$ band.

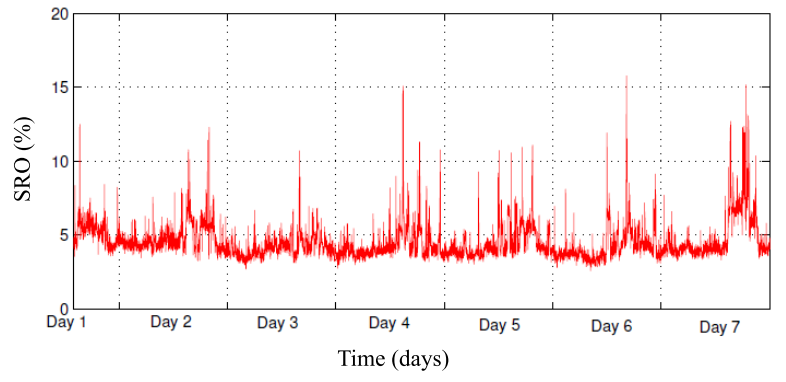

(c) SRO for the entire $2.35-2.50 \mathrm{GHz}$ band.

Fig. 6. CO and SRO for the entire $2.35-2.50 \mathrm{GHz}$ band over the whole week Second measurement campaign (accident and emergency ward).

\section{A. Time-frequency statistical model of the interference}

The data set measured in the daily surgery ward was chosen to determine the time-frequency distribution of the aggregate interference in different interference regimes. ${ }^{4}$ We consider several candidate distributions including: Burr; exponential; extreme value; Gaussian; generalized extreme value (GEV); logistic; log-normal; t-location scale; and Weibull. The selection is performed using an algorithm developed based on [35]. Table II shows the selected distribution and the associated parameters for different scenarios in the delay surgery ward. The four best fit distributions are listed in the following, where $x$ is a RV representing the received power in time and frequency.

\footnotetext{
${ }^{4}$ The daily surgery data set was chosen since it was the largest one containing the full dynamic range of the interference, and it has the same interference characteristics as of the other two wards.
}

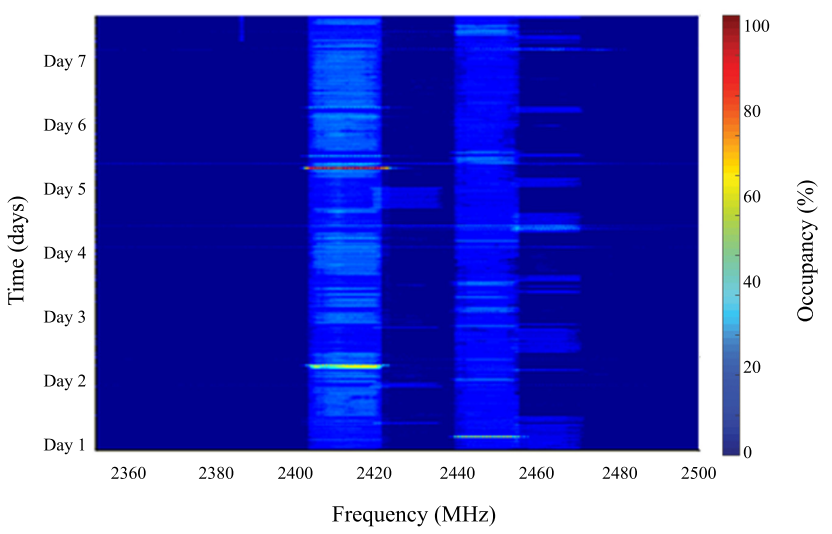

Fig. 7. CO for the entire $2.35-2.50 \mathrm{GHz}$ band over the whole week. Third measurement campaign (X-ray and radiology ward).

The GEV distribution [36] is given by

$$
\begin{aligned}
f_{\mathrm{\times}}(x \mid k, \mu, \sigma)=\frac{1}{\sigma} \exp \{ & \left.-\left(1+k \frac{x-\mu}{\sigma}\right)^{-1 / k}\right\} \\
& \times\left(1+k \frac{x-\mu}{\sigma}\right)^{-1-1 / k}
\end{aligned}
$$

where $k$ is the shape parameter, $\mu$ is the location parameter, and $\sigma$ is the scale parameter.

The Burr type XII distribution [36] is given by

$$
f_{\mathrm{\times}}(x \mid \alpha, c, k)=\frac{k c(x / \alpha)^{c-1}}{\alpha\left(1+(x / \alpha)^{c}\right)^{k+1}}
$$

where $c$ and $k$ are the shape parameters, and $\alpha$ is the scale parameter.

The t-location scale distribution [36] is given by

$$
f_{\mathrm{x}}(x \mid \mu, \sigma, \nu)=\frac{\Gamma\left(\frac{\nu+1}{2}\right)}{\sigma \sqrt{\pi \nu} \Gamma(\nu / 2)}\left(\frac{\nu+\frac{(x-\mu)^{2}}{\sigma}}{\nu}\right)^{-\frac{\nu+1}{2}}
$$

where $\Gamma(\cdot)$ represents the gamma function, $\mu$ is the location parameter, $\sigma$ is the scale parameter, and $\nu$ is the shape parameter.

The logistic distribution [36] is given by

$$
f_{\mathrm{x}}(x \mid \mu, \sigma)=\frac{e^{\frac{x-\mu}{\sigma}}}{\sigma\left(1+e^{-\frac{x-\mu}{\sigma}}\right)^{2}}
$$

where $\mu$ is the location parameter and $\sigma$ is the scale parameter.

We further consider two scenarios of interest, namely low and high interference, for the popular IEEE $802.11 \mathrm{~b} / \mathrm{g}$ systems in the daily surgery ward. It was found that the stable distribution is the best fit for these two scenarios. The characteristic function of a stable distribution [36] is given by

$$
\Phi_{\times}(x \mid \alpha, \beta, \gamma, \delta)=\exp \left\{\imath x \gamma-|\delta x|^{\alpha}(1-\imath \beta \operatorname{sgn}(x) \phi)\right\}
$$

where $x$ is a RV representing the received power in time and frequency, $\imath$ is the imaginary unit, $\alpha$ is the first shape parameter, $\beta$ is the second shape parameter, $\gamma$ is the scale parameter, and $\delta$ is the location parameter, and

$$
\phi= \begin{cases}\tan (\pi \alpha / 2) & \text { for } \alpha \neq 1 \\ -(2 / \pi) \log |x| & \text { for } \alpha=1\end{cases}
$$


TABLE II

SELECTED DISTRIBUTIONS AND ASSOCIATED PARAMETERS.

\begin{tabular}{|l|l|l|}
\hline Scenario & Selected Distribution & Distribution Parameters \\
\hline Day 1 Spectrum Variation & GEV & $k=0.6676 ; \sigma=2.9557 ; \mu=-85.9427$ \\
Day 2 Spectrum Variation & GEV & $k=0.6370 ; \sigma=2.8504 ; \mu=-85.5746$ \\
Day 3 Spectrum Variation & GEV & $k=0.7941 ; \sigma=2.8023 ; \mu=-85.7233$ \\
Day 4 Spectrum Variation & GEV & $k=0.8568 ; \sigma=2.6080 ; \mu=-85.9357$ \\
Day 5 Spectrum Variation & GEV & $k=0.7900 ; \sigma=2.7385 ; \mu=-85.8011$ \\
Day 6 Spectrum Variation & GEV & $k=0.8520 ; \sigma=3.0775 ; \mu=-85.6074$ \\
Day 7 Spectrum Variation & GEV & $k=0.7812 ; \sigma=2.9172 ; \mu=-85.6607$ \\
Whole Week Spectrum Variation & GEV & $k=0.7812 ; \sigma=2.8348 ; \mu=-85.9843$ \\
Whole Week IEEE 802.11b/g Ch. 1 & Burr type XII & $\alpha=5.7691 ; c=43.5808 ; k=0.2567$ \\
Whole Week IEEE 802.11b/g Ch. 6 & t-location scale & $\mu=5.3937 ; \sigma=0.3650 ; \nu=1.9946$ \\
Whole Week IEEE 802.11b/g Ch. 11 & logistic & $\mu=-0.0094 ; \sigma=0.0290$ \\
Whole Week IEEE 802.11n Ch. 3 & GEV & $k=0.2759 ; \sigma=0.0380 ; \mu=0.0711$ \\
Whole Week IEEE 802.11n Ch. 9 & logistic & $\mu=0.0563 ; \sigma=0.0047$ \\
Whole Week FBO Variation & t-location scale & $\mu=0.8664 ; \sigma=0.0360 ; \nu=2.4698$ \\
Whole Week SRO (IEEE 802.11b/g) & GEV & $k=0.4101 ; \sigma=0.0022 ; \mu=0.0055$ \\
Whole Week SRO (IEEE 802.11n) & t-location scale & $\mu=3.8262 ; \sigma=0.1370 ; \nu=2.2628$ \\
\hline
\end{tabular}

TABLE III

BIC AND JSD VALUES FOR HIGH AND LOW INTERFERENCE SCENARIOS.

\begin{tabular}{|c|c|c|c|}
\hline Scenario & Distribution & BIC & JSD \\
\hline Low & stable & $3.0928 \times 10^{4}$ & $7.5753 \times 10^{-4}$ \\
& logistic & $3.1005 \times 10^{4}$ & $8.0385 \times 10^{-4}$ \\
& t-location scale & $3.1060 \times 10^{4}$ & $8.0602 \times 10^{-4}$ \\
& Gaussian & $3.1217 \times 10^{4}$ & $7.6444 \times 10^{-4}$ \\
& GEV & $3.1795 \times 10^{4}$ & $1.0131 \times 10^{-3}$ \\
& extreme value & $3.5839 \times 10^{4}$ & $2.2964 \times 10^{-3}$ \\
\hline High & stable & $3.4938 \times 10^{4}$ & $1.7755 \times 10^{-3}$ \\
& Burr type XII & $3.4959 \times 10^{4}$ & $1.9803 \times 10^{-3}$ \\
& GEV & $3.4963 \times 10^{4}$ & $2.0030 \times 10^{-3}$ \\
& t-location scale & $3.5099 \times 10^{4}$ & $2.0984 \times 10^{-3}$ \\
& logistic & $3.5131 \times 10^{4}$ & $1.9824 \times 10^{-3}$ \\
& Gaussian & $3.5425 \times 10^{4}$ & $1.9814 \times 10^{-3}$ \\
\hline
\end{tabular}

These results agree with the theory of statistical distribution for the aggregate interference developed in [4]-[6], [37]-[42].

Table III shows the BIC and JSD values for the six best statistical distributions in low and high interference scenarios, for the popular IEEE $802.11 \mathrm{~b} / \mathrm{g}$ systems in the daily surgery ward. The stable distribution is always the best fit distribution to the empirical data. The parameters of the best fitting (Stable) distribution are summarized in Table IV.

\section{B. Cluster-based statistical model of the interference}

In this section, a cluster-based statistical model ${ }^{5}$ of the aggregate interference is developed for two scenarios of interest, namely low and high interference, for the popular IEEE $802.11 \mathrm{~b} / \mathrm{g}$ systems over all the three measurement campaigns. In particular, channel $6(2.437 \mathrm{GHz})$ showed low

\footnotetext{
${ }^{5}$ For a given channel, a cluster is defined as a group of consecutive samples above the threshold.
}

interference in the X-ray and radiology ward, while channel 1 $(2.412 \mathrm{GHz})$ showed high interference in the accident and emergency ward. The cluster-based statistical model relies on the distributions of cluster size, cluster interference sample, and cluster inter-arrival time. For a given channel: the cluster size is the cardinality of a cluster; the cluster interference sample is the value of a sample inside a cluster; and the cluster inter-arrival time is the difference between the first sweep index of a sample in a cluster and the last sweep index of a sample in the previous cluster.

Fig. 8 shows the empirical probability mass function (PMF) of the cluster size. It can be observed that the low interference scenario exhibits a smaller variability of the cluster size compared to the high interference scenario. In particular, the maximum cluster size is 14 and 79 samples in the low and high interference scenarios, respectively. The best fit distribution for the cluster size in both low and high interference scenarios is geometric [36] given by

$$
f_{\mathrm{x}}(x \mid p)=p(1-p)^{x-1}
$$

where $\mathrm{x}$ is a RV representing the cluster size for the specific channel and $p$ is the success probability parameter. The associated parameter is $p=0.9755$ for the low interference scenario, and is $p=0.8896$ for the high interference scenario.

Fig. 9 shows the empirical PDF of the cluster interference sample. It can be observed that the cluster interference samples range from -84 to $-19 \mathrm{dBm}$ and from -84 to $-17 \mathrm{dBm}$ in the low and high interference scenarios, respectively. While the ranges are similar in the two scenarios, we observed from our data that in the high interference scenario, there are about 30 times larger number of samples occupying the channel than in the low interference scenario. Note that the empirical PDFs are obtained by normalizing over such numbers of samples.The best fit distribution for cluster interference sample in both low and high interference scenarios is GEV given by (14), where $x$ is a RV representing the cluster interference sample for the 


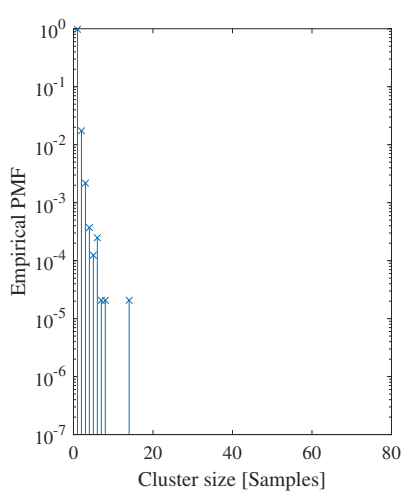

(a) Low Interference.

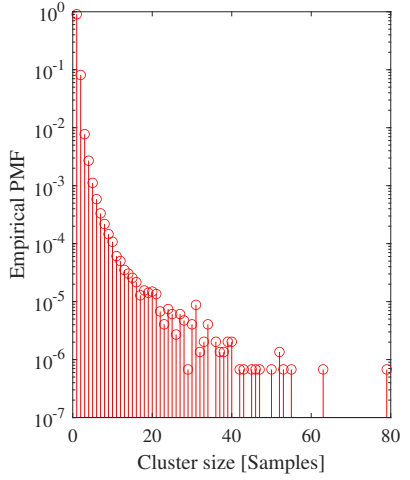

(b) High Interference.
Fig. 8. Empirical PMF of the cluster size in low and high interference scenarios.

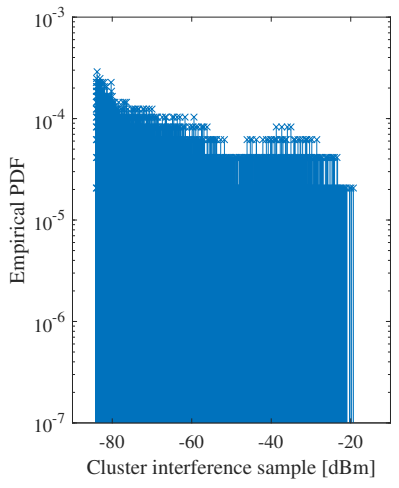

(a) Low Interference.

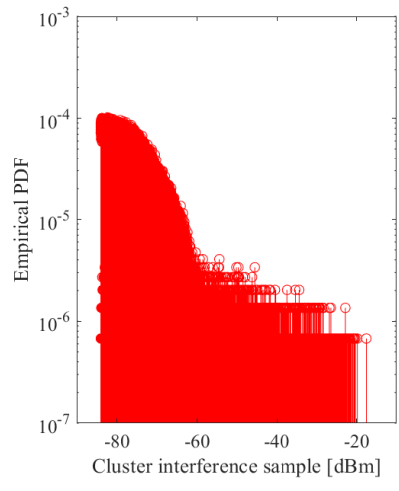

(b) High Interference.
Fig. 9. Empirical PDF of the cluster interference sample in low and high interference scenarios.

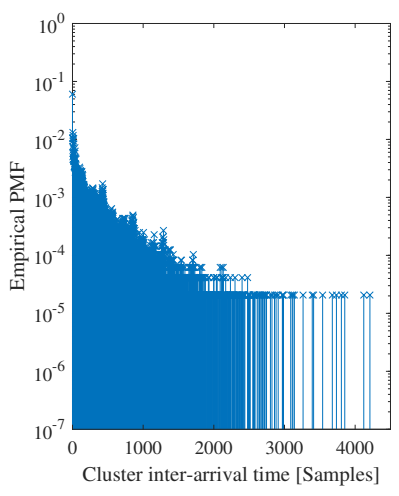

(a) Low Interference.

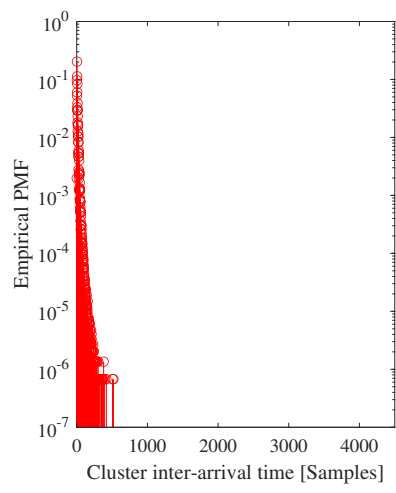

(b) High Interference.
Fig. 10. Empirical PMF of the cluster inter-arrival time in low and high interference scenarios.

specific channel. The associated parameters are $k=0.65$, $\sigma=6.30$, and $\mu=-78.41$ for the low interference scenario, and are $k=0.12, \sigma=3.73$, and $\mu=-79.44$ for the high interference scenario.

Fig. 10 shows the empirical PMF of the cluster inter-arrival time. It can be observed that the low interference scenario
TABLE IV

PARAMETERS OF THE STABLE DISTRIBUTION FOR THE LOW AND HIGH INTERFERENCE SCENARIOS.

\begin{tabular}{|c|c|r|c|c|}
\hline & $\alpha$ & \multicolumn{1}{c|}{$\beta$} & $\gamma$ & $\delta$ \\
\hline Low & 1.818 & -0.938 & 2.278 & -94.755 \\
High & 1.707 & 0.934 & 4.967 & -96.550 \\
\hline
\end{tabular}

TABLE V

SIMILARITY BETWEEN THE EMPIRICAL DISTRIBUTIONS OF GENERATED DATA AND OF MEASURED DATA.

\begin{tabular}{|c|c|}
\hline Scenario & JSD \\
\hline Low & $6.0594 \times 10^{-4}$ \\
High & $1.8858 \times 10^{-6}$ \\
\hline
\end{tabular}

exhibits a larger variability of the cluster inter-arrival time compared to the high interference scenario. In particular, the maximum inter-arrival time is around 4200 and 520 samples in the low and high interference scenarios, respectively. The best fit distribution for the cluster inter-arrival time in both low and high interference scenarios is geometric [36] given by (20), where $\mathrm{x}$ is a RV representing the cluster inter-arrival time for the specific channel and $p$ is the success probability parameter. The associated parameter is $p=0.0037$ for the low interference scenario, and is $p=0.1027$ for the high interference scenario.

The similarity between the empirical distributions (of data generated from the cluster-based statistical model and of data gathered from measurements) is quantified using the JSD in (12). In particular, Table V shows the JSD values for the low and high interference scenarios.

\section{CONCLUSION}

This paper characterized the aggregate interference in an hospital environment for the largely used $2.35-2.50 \mathrm{GHz}$ band based on a network experimentation representing three oneweek-long measurement campaigns. Leveraging on these measurements, the spectrum occupancy is quantified according to three metrics, namely CO, FBO and SRO. Two statistical models of the aggregate interference have been proposed: the first model describes the aggregate interference (plus noise) over the entire time-frequency intervals; while the second model describes the aggregate interference (without noise) by grouping the interference samples in clusters. Our results show that mission-critical medical services in the ISM band can be severely hampered by the interference, calling for the use of reserved sub-bands or the use of interference detection and mitigation techniques. The proposed models also serve as basis for simulating network behavior and designing medical devices in wireless hospital environments.

\section{ACKNOWLEDGMENT}

The authors wish to thank the Editor S. Pollin, the anonymous Reviewers, and R. Cohen for helpful suggestions and careful reading of the paper. The authors also wish to thank the Oulu University Hospital for providing access to their wards during the network experimentations. 


\section{REFERENCES}

[1] V. Valenta, R. Maršálek, G. Baudoin, M. Villegas, M. Suarez, and F. Robert, "Survey on spectrum utilization in Europe: Measurements, analyses and observations," in Proc. IEEE Int. Conf. on Cognitive Radio Oriented Wireless Networks and Commun., Jun. 2010, pp. 1-5.

[2] A. D. Spaulding and G. H. Hagn, "On the definition and estimation of spectrum occupancy," IEEE Trans. Electromagn. Compat., vol. EMC-19, no. 3, pp. 269-280, Aug. 1977.

[3] A. Conti, B. M. Masini, F. Zabini, and O. Andrisano, "On the down-link performance of multi-carrier CDMA systems with partial equalization," IEEE Trans. Wireless Commun., vol. 6, no. 1, pp. 230-239, Jan. 2007.

[4] M. Z. Win, P. C. Pinto, A. Giorgetti, M. Chiani, and L. A. Shepp, "Error performance of ultrawideband systems in a Poisson field of narrowband interferers," in Proc. IEEE Int. Symp. on Spread Spectrum Techn. \& Applicat., Manaus, Brazil, Aug. 2006, pp. 410-416.

[5] M. Z. Win, "A mathematical model for network interference," IEEE Communication Theory Workshop, Sedona, AZ, May 2007.

[6] M. Z. Win, P. C. Pinto, and L. A. Shepp, "A mathematical theory of network interference and its applications," Proc. IEEE, vol. 97, no. 2 , pp. 205-230, Feb. 2009, special issue on Ultra-Wide Bandwidth (UWB) Technology \& Emerging Applications.

[7] A. Conti, D. Dardari, G. Pasolini, and O. Andrisano, "Bluetooth and IEEE $802.11 \mathrm{~b}$ coexistence: Analytical performance evaluation in fading channels," IEEE J. Sel. Areas Commun., vol. 21, no. 2, pp. 259-269, Feb. 2003.

[8] A. Rabbachin, T. Q. Quek, H. Shin, and M. Z. Win, "Cognitive network interference," IEEE J. Sel. Areas Commun., vol. 29, no. 2, pp. 480-493, Feb. 2011.

[9] F. Zabini, A. Bazzi, B. M. Masini, and R. Verdone, "Optimal performance versus fairness tradeoff for resource allocation in wireless systems," IEEE Trans. Wireless Commun., vol. 16, no. 4, pp. 25872600, Apr. 2017.

[10] F. Zabini, A. Bazzi, and B. M. Masini, "Throughput versus fairness tradeoff analysis," in Proc. IEEE Int. Conf. Commun., Jun. 2013, pp. 5131-5136.

[11] J. Kokkoniemi and J. J. Lehtomäki, "Spectrum occupancy measurements and analysis methods on the $2.45 \mathrm{GHz}$ ISM band," in Proc. IEEE Int. Conf. on Cognitive Radio Oriented Wireless Networks and Commun., Jun. 2012, pp. 285-290.

[12] T. Keller and J. Modelski, "Experimental results of testing interferences in $2.4 \mathrm{GHz}$ ISM band," in Proc. European Microw. Conf., Oct. 2003, pp. 1043-1046.

[13] T. Wollenberg, S. Bader, and A. Ahrens, "Measuring channel occupancy for 802.11 wireless LAN in the $2.4 \mathrm{GHz}$ ISM band," in Proc. ACM Int. Conf. on Model., Anal. and Simul. of Wireless and Mobile Syst., Oct. 2012, pp. 305-308.

[14] J.-A. Park, S.-K. Park, D.-H. Kim, P.-D. Cho, and K.-R. Cho, "Experiments on radio interference between wireless LAN and other radio devices on a $2.4 \mathrm{GHz}$ ISM band," in Proc. IEEE Semiannual Veh. Technol. Conf., vol. 3, Apr. 2003, pp. 1798-1801.

[15] K.-S. Tan, I. Hinberg, and J. Wadhwani, "Electromagnetic interference in medical devices: Health Canada's past and current perspectives and activities," in Proc. IEEE Int. Symp. on Electromagn. Compat., vol. 2, Aug. 2001, pp. 1283-1288.

[16] S. Krishnamoorthy, J. H. Reed, C. R. Anderson, P. M. Robert, and S. Srikanteswara, "Characterization of the $2.4 \mathrm{GHz}$ ISM band electromagnetic interference in a hospital environment," in Proc. IEEE Int. Conf. Eng. Medicine and Biol. Soc., vol. 4, Sept 2003, pp. 3245-3248.

[17] M. O. A. Kalaa, G. Butron, W. Balid, H. H. Refai, and N. J. LaSorte, "Long term spectrum survey of the $2.4 \mathrm{GHz}$ ISM band in multiple hospital environments," in Proc. IEEE Wireless Commun. and Netw. Conf., Apr. 2016, pp. 246-251.

[18] "Smart body area network (SmartBAN): Measurements and modelling of SmartBAN radio frequency (RF) environment," http://www.etsi.org/deliver/etsi_tr/103300_103399/103395/01.01.01 60/tr_103395v010101p.pdf, European Telecommunications Standards Institute (ETSI), TR 103 395, Dec 2016.

[19] H. Viittala, L. Mucchi, M. Hämäläinen, and T. Paso, "ETSI SmartBAN system performance and coexistence verification for healthcare," IEEE Access, vol. 5, pp. 8175-8182, 2017.

[20] M. H. Virk, R. Vuohtoniemi, M. Hämäläinen, J. Iinatti, and J. P. Mäkelä, "On spectrum occupancy evaluations from the standpoint of body area networks in ISM band," in Int. Symp. on Med. Inf. and Commun. Technol., Mar. 2015, pp. 15-19.
[21] L. Mucchi, A. Carpini, T. D’Anna, M. H. Virk, R. Vuohtoniemi, M. Hämäläinen, and J. Iinatti, "Threshold setting for the evaluation of the aggregate interference in ISM band in hospital environments," in Int. Symp. on Med. Inf. and Commun. Technol., Mar. 2015, pp. 20-24.

[22] T. D'Anna, "Evaluation of the aggregate interference in $2.4 \mathrm{GHz}$ ISM band in home, office and hospital environments," Master's thesis, University of Oulu, 2015.

[23] M. H. Virk, R. Vuohtoniemi, M. Hämäläinen, J.-P. Mäkelä, and J. Iinatti, "Spectrum occupancy evaluations at $2.35-2.50 \mathrm{GHz}$ ISM band in a hospital environment," in Proc. Int. Conf. on Body Area Netw., Sep. 2014, pp. 39-45.

[24] Handbook on Spectrum Monitoring, http://www.itu.int/pub/ R-HDB-23-2011, International Telecommunication Union (ITU), Radiocommunication (ITU-R), 2011.

[25] "Impact of industrial, scientific and medical (ISM) equipment on radiocommunication services," http://www.itu.int/pub/R-REP-SM.2180, International Telecommunication Union (ITU), Report SM.2180, Tech. Rep., 2010.

[26] "Spectrum occupancy measurements and evaluation," http://www.itu.int/ pub/R-REP-SM.2256/ar, International Telecommunication Union (ITU) Report SM.2256-1, Tech. Rep., 2016.

[27] K. Patil, R. Prasad, and K. Skouby, "A survey of worldwide spectrum occupancy measurement campaigns for cognitive radio," in Proc. Int. Conf. on Devices and Commun., Feb. 2011, pp. 1-5.

[28] J. J. Lehtomäki, R. Vuohtoniemi, K. Umebayashi, and J.-P. Mäkelä, "Energy detection based estimation of channel occupancy rate with adaptive noise estimation," IEICE Trans. Commun., vol. E95.B, no. 4, pp. 1076-1084, Apr. 2012

[29] P. Henttu and S. Aromaa, "Consecutive mean excision algorithm," in Proc. IEEE Int. Symp. on Spread Spectrum Techn. \& Applicat., vol. 2 Sep. 2002, pp. 450-454.

[30] "Oulu university hospital description," https://www.ppshp.fi/en/Pages/ default.aspx.

[31] ARA, "Data sheet for cma-118/a antenna," http://www.ic72.com/pdf_ file/d/103797.pdf.

[32] M. H. Virk, R. Vuohtoniemi, M. Hämäläinen, J. Iinatti, and J. P Mäkelä, "Stochastic spectral occupancy modeling: A body area network perspective in ISM band," in Int. Symp. on Med. Inf. and Commun. Technol., Mar. 2015, pp. 157-161.

[33] G. E. P. Box, G. M. Jenkins, G. C. Reinsel, and G. M. Ljung, Time Series Analysis: Forecasting and Control, 5th ed. Wiley, 2015.

[34] J. Lin, "Divergence measures based on the Shannon entropy," IEEE Trans. Inf. Theory, vol. 37, no. 1, pp. 145-151, Jan. 1991.

[35] M. Sheppard, https://it.mathworks.com/matlabcentral/fileexchange/ 36000-fbd-find-the-best-distribution-tool, MIT Lincoln Laboratory.

[36] K. Krishnamoorthy, Handbook of Statistical Distributions with Applications, 2nd ed. Boca Raton, FL: Chapman and Hall/CRC, 2016.

[37] P. C. Pinto and M. Z. Win, "Communication in a Poisson field of interferers - Part I: Interference distribution and error probability," IEEE Trans. Wireless Commun., vol. 9, no. 7, pp. 2176-2186, Jul. 2010.

[38] — "Communication in a Poisson field of interferers - Part II: Channel capacity and interference spectrum," IEEE Trans. Wireless Commun., vol. 9, no. 7, pp. 2187-2195, Jul. 2010.

[39] P. C. Pinto, A. Giorgetti, M. Z. Win, and M. Chiani, "A stochastic geometry approach to coexistence in heterogeneous wireless networks," IEEE J. Sel. Areas Commun., vol. 27, no. 7, pp. 1268-1282, Sep. 2009, special issue on Stochastic Geometry and Random Graphs for Wireless Networks.

[40] A. Rabbachin, A. Conti, and M. Z. Win, "Wireless network intrinsic secrecy," IEEE/ACM Trans. Netw., vol. 23, no. 1, pp. 56 - 69, Feb. 2015.

[41] M. Z. Win, A. Rabbachin, J. Lee, and A. Conti, "Cognitive network secrecy with interference engineering," IEEE Netw., vol. 28, no. 5, pp. 86 - 90, Sep./Oct. 2014.

[42] H. ElSawy, A. Sultan-Salem, M.-S. Alouini, and M. Z. Win, "Modeling and analysis of cellular networks using stochastic geometry: A tutorial," IEEE Commun. Surveys Tuts., vol. 19, no. 1, pp. 167-203, First Quarter 2017. 


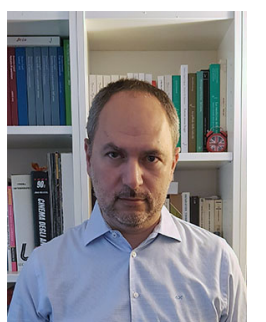

Lorenzo Mucchi (M'98-SM'12) is an Associate Professor at the Department of Information Engineering of the University of Florence, Italy. He is the founder and scientific coordinator of two Joint Research Laboratories: one on Visible Light Communications and another on Medical ICTs.

In 2000, he was a visiting researcher at the Centre for Wireless Communications of the University of Oulu, Finland. His research interests involve theory and experimentation of wireless systems and networks including physical-layer security, visible light communications, localization and tracking, body area networks, and molecular communications.

Dr. Mucchi is serving as an Associate Editor of IEEE Communications Letter and IEEE Access, and he has been Editor-in-Chief for Elsevier Academic Press. Since 2013, he has been a member of the Smart Body Area Network group in the European Telecommunications Standard Institute (ETSI), where he lead items on security, coexistence, and interference mitigation. He chaired IEEE and EAI international conferences.

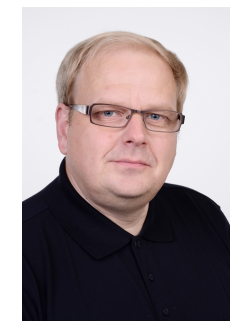

Risto Vuohtoniemi received the M.Sc. (Tech) and the Lic.Tech. degrees in electrical engineering from the University of Oulu, Finland. He is currently a University Teacher with the Faculty of Information Technology and Electrical Engineering, University of Oulu and a Research Scientist with the Centre for Wireless Communications (CWC) at the University of Oulu. His research interests are in RF technology, especially in future wireless communication systems, cognitive radio, power line communication and passive radar applications. He was a recipient of the Best Paper Award in IEEE VTC 2019-Fall, as a co-author. He has served as a Technical Program Committee Member for numerous IEEE conferences.

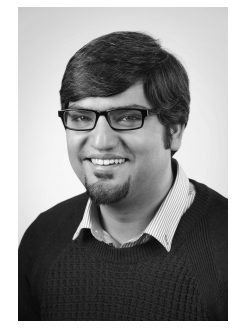

Hasnain Virk received his M.Sc degree in Wireless Communication Engineering from University of Oulu, Finland in 2013. Currently he is working as an Expert Software Architect at Elektrobit Automotive Finland developing secure communication stack for fully connected Electric Vehicles. He worked as a Senior IoT Specialist at Arm from 2015 to 2019 and was part of the team that developed an open source RTOS (Mbed OS) for constrained IoT devices. He actively participated and contributed to the LoRaWAN standardisation and was elected Chairman of the LoRa Alliance Developer Community WG in 2018. Prior to that Hasnain worked as a Research Scientist at Centre for Wireless Communications (CWC), University of Oulu where he worked on Wireless Body Area Networks (WBAN) and developed mathematical models for interference in ISM bands specifically in hospital environments. His research interests are IoT, data analytics and climate change.

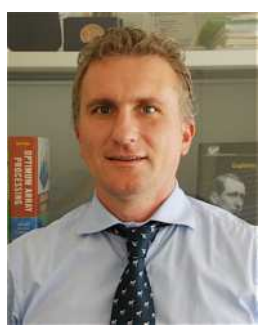

Andrea Conti (S'99-M'01-SM'11) is a Professor and founding director of the Wireless Communication and Localization Networks Laboratory at the University of Ferrara, Italy. Prior to joining the University of Ferrara, he was with the CNIT and with the IEIIT-CNR.

In Summer 2001, he was with the Wireless Systems Research Department at AT\&T Research Laboratories. Since 2003, he has been a frequent visitor to the Wireless Information and Network Sciences Laboratory at the Massachusetts Institute of Technology, where he presently holds the Research Affiliate appointment. His research interests involve theory and experimentation of wireless communication and localization. His current research topics include network localization and navigation, distributed sensing, adaptive diversity communications, and quantum information science.

Dr. Conti has served as editor for IEEE journals and chaired international conferences. He was elected Chair of the IEEE Communications Society's Radio Communications Technical Committee and is Co-founder of the IEEE Quantum Communications \& Information Technology Emerging Technical Subcommittee. He received the HTE Puskás Tivadar Medal, the IEEE Communications Society's Fred W. Ellersick Prize, and the IEEE Communications Society's Stephen O. Rice Prize in the field of Communications Theory. He is an elected Fellow of the IET and a member of Sigma Xi. He has been selected as an IEEE Distinguished Lecturer.

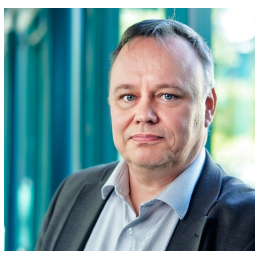

Matti Hämäläinen (SM'09) received his M.Sc, Lic.Tech. and Dr.Sc. degrees in 1994, 2002 and 2006, respectively, from University of Oulu, Finland. Currently he is an Adjunct Professor and University Researcher at Centre for Wireless Communications, University of Oulu. He was nominated as an IAS Visiting Professor at Yokohama National University, Japan, during 2016-2018. His research interests are in ultra wideband systems, radio channel modeling, wireless body area networks and medical ICT. He has more than 150 scientific publications and holds one patent. He is a co-editor of three books, and co-author of one book and two book chapters. He is an Associate Editor of IEEE Access, member of Editorial Board of Annals of Telecommunications, serving as a reviewer for IEEE and IET journals. He has been a Technical Program Committee Member for numerous IEEE conferences and was a General Chair of Bodynets'2018. He is also Co-Chair of International Steering Committee of ISMICT conference. Dr. Hämäläinen is an active member of the European Telecommunications Standard Institute (ETSI) Smart Body Area Network (SmartBAN) technical committee.

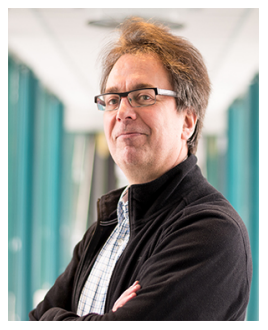

Jari Iinatti received M.Sc., Lic. Tech. and Dr. Tech. degrees in electrical engineering from the University of Oulu, Oulu, Finland, in 1989, 1993 and 1997, respectively. During 1989-1997 he was a Research Scientist at the Telecommunication Laboratory at the University of Oulu, during 1997-2002 acting professor of Digital Transmission Techniques at Centre for Wireless Communications (CWC) at the University of Oulu. Since 2002 he has been Professor of Telecommunication Theory, and is currently Head of CWC - Networks and Systems as well as Dean of Education at the Faculty of Information Technology and Electrical Engineering. He was IAS Visiting Professor at Yokohama National University, Japan, during 2016-2018. His research interests include future wireless communication systems, transceiver algorithms and medical ICT. Prof. Iinatti has published around 250 International Journal and Conference papers and holds six patents. He has been technical program committee (TPC) member in about 25 conferences, and TPC co-chair, TPC chair, general co-chair and TPC Program Track Co-Chair in several conferences. He serves as Steering Committee Co-Chair of ISMICT series.

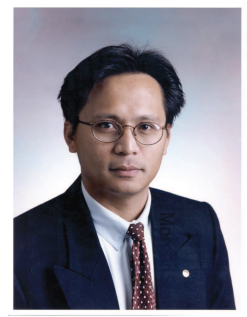

Moe Z. Win (S'85-M'87-SM'97-F'04) is a Professor at the Massachusetts Institute of Technology (MIT) and the founding director of the Wireless Information and Network Sciences Laboratory. Prior to joining MIT, he was with AT\&T Research Laboratories and NASA Jet Propulsion Laboratory.

His research encompasses fundamental theories, algorithm design, and network experimentation for a broad range of real-world problems. His current research topics include network localization and navigation, network interference exploitation, and quantum information science. He has served the IEEE Communications Society as an elected Member-at-Large on the Board of Governors, as elected Chair of the Radio Communications Committee, and as an IEEE Distinguished Lecturer. Over the last two decades, he held various editorial positions for IEEE journals and organized numerous international conferences. Recently, he has served on the SIAM Diversity Advisory Committee.

Dr. Win is an elected Fellow of the AAAS, the EURASIP, the IEEE, and the IET. He was honored with two IEEE Technical Field Awards: the IEEE Kiyo Tomiyasu Award (2011) and the IEEE Eric E. Sumner Award (2006, jointly with R. A. Scholtz). His publications, co-authored with students and colleagues, have received several awards. Other recognitions include the IEEE Communications Society Edwin H. Armstrong Achievement Award (2016), the International Prize for Communications Cristoforo Colombo (2013), the Copernicus Fellowship (2011) and the Laurea Honoris Causa (2008) from the Università degli Studi di Ferrara, and the U.S. Presidential Early Career Award for Scientists and Engineers (2004). He is an ISI Highly Cited Researcher. 